\title{
Alter
}

Revue de phénoménologie

19 | 2011

Le langage

\section{L'éloquence « de » la première personne}

\section{Natalie Depraz}

\section{OpenEdition}

Journals

Édition électronique

URL : http://journals.openedition.org/alter/1365

DOI : $10.4000 /$ alter.1365

ISSN : 2558-7927

\section{Éditeur :}

Association ALTER, Archives Husserl (CNRS-UMR 8547)

\section{Édition imprimée}

Date de publication : 1 octobre 2011

Pagination : $57-64$

ISBN : 978-2-9522374-7-5

ISSN : 1249-8947

Référence électronique

Natalie Depraz, «L'éloquence « de » la première personne », Alter [En ligne], 19 | 2011, mis en ligne le 01 janvier 2020, consulté le 23 janvier 2020. URL : http://journals.openedition.org/alter/1365 ; DOI : 10.4000/alter.1365 


\section{L'ÉLOQUENCE «DE » LA PREMIÈRE PERSONNE (SÉMINAIRE « ALTER » 2009-2010 «LES CHOSES ET LES MOTS ", JOURNÉE DU 12 JUIN 2010)}

Natalie Depraz

\section{Introduction}

J'ai choisi ce titre à la fois énigmatique et évocateur pour attirer votre attention sur un point très précis à l'intérieur de la relation macroscopique entre le langage et l'expérience dans les deux traditions philosophiques contemporaines, la phénoménologie et la philosophie analytique, lesquelles sont souvent appréhendées comme antinomiques: il s'agira ici de s'interroger sur le langage très particulier que requiert une attitude en première personne; on verra que cette dernière implique très souvent (mais pas nécessairement) le pronom personnel «Je», et que - le fil conducteur que j'ai choisi l'indique d'emblée assez clairement - elle n'est en rien, le plus souvent, de l'ordre de l'ineffable, de l'indicible, même si elle peut aussi se manifester parfois sous le régime du silence. Bref, ici, mon fil conducteur : "l'éloquence de la première personne », cherche à faire ressortir la force du langage en jeu (son éloquence : sa significativité, la force de son sens) et le fait que c'est la première personne (en tant que génétif subjectif) qui est éloquente, que c'est elle en tant qu'elle est ce qu'elle est qui est langage, qui a son langage propre, qu'elle n'est donc pas le simple « objet » (au sens du thème) d'un langage qui $\mathrm{s}^{\prime} \mathrm{y}$ appliquerait (selon le sens du génitif objectif).

Par cette exploration, je cherche une voie d'entrée dans cette question énorme de la relation entre langage et expérience au sein des deux traditions sus-dites, qui permette de se situer à l'écart des options un peu trop radicales en présence : d'une part, en phénoménologie, conformément au mot d'ordre husserlien d'un retour aux 
choses elles-mêmes, l'expérience «muette encore » qui vient d'ellemême, sui generis, à « l'expression de son propre sens »; d'autre part, en philosophie dite "analytique », conformément, par exemple, au mot d'ordre wittgensteinien selon lequel «philosopher, c'est résister à la fascination qu'exercent sur nous certaines formes d'expression ", le langage situationnel a-subjectif, i.e. dénué d'affect de la logique, puis, plus tard, de l'éthique sobre du quotidien.

Certes, le bilan n'est pas aussi manichéen, les phénoménologues s'interrogent sur le mode d'expression des phénomènes, bien enten$\mathrm{du}$ (Husserl, quoique sa position reste ambivalente, Heidegger avec un souci de défaire l'emprise du logique au profit de l'être, MerleauPonty exemplairement, mais avec un souci tel d'immanence de l'expression qu'elle en devient paradoxalement infra-langagière, Michel Henry sur un mode polémique extrêmement virulent à l'encontre de l'herméneutique du logos, Derrida avec sa critique du logocentrisme); les philosophes analytiques de leur côté interrogent également le contexte expérientiel et la situation intersubjective dans laquelle s'inscrit le langage: c'est le cas, de façon exemplaire, du dernier Wittgenstein, où la recherche d'un langage ordinaire prend sa source dans un intérêt pour une expression "monstrative » plus que démonstrative, qui fait des mots situationnels, les indicateurs de lieu et de temps, des supports expérientiels ; plus en amont, les pragmatistes Austin puis Searle mettent au premier plan, dans le langage, l'énonciation, i.e. le contexte illocutoire et perlocutoire de la prise de parole, à savoir l'impact interpersonnel et social du langage.

Il y a donc bien des croisements, des recherches actuelles situées au confluent des deux traditions (Husserl/Wittgenstein ; Benvéniste et la phénoménologie; Ricœur et la philosophie analytique de l'action), et mon propos se situe sur le fond de ces recherches croisées. Mais je voudrais, pour commencer à mieux cibler ma perspective, pratiquer deux distinctions méthodiques, concernant, d'une part, ce que j'entends par " phénoménologie », d'autre part, ce que je mets sous le terme « langage ».

Par «phénoménologie », on entend le plus souvent une approche qui met au premier plan l'expérience et cherche à désencombrer les concepts de leur gangue logiquement cohérente et auto-référentielle ; on fait le pari que le phénoménologue «vit »ce qu'il écrit, y est présent de façon incarnée, ce qui fait la différence avec une approche philosophique formelle. Pour autant, l'identification assez fréquente entre phénoménologie et approche en première personne (D. Carr, D. Lohmar, D. Zahavi, J.-L. Marion) est loin d'aller de soi. En effet, la plupart du temps, l'exigence d'une telle relation incarnée à l'expérience ne se traduit pas dans les faits, c'est-à-dire qu'elle se traduit en 
réalité par un propos qui reste générique, peu en contact avec une expérience singulière, où l'instance subjective, lorsqu'elle est mobilisée, demeure une entité principielle non-située (le Je transcendantal, le Dasein, le visage, l'autre, le soi), en surplomb, en extériorité, où les exemples qui pourraient ancrer le propos dans un vécu irréductible à tout autre et, ce faisant, incarné, restent donnés en général ou de façon hypothétique, comme s'ils pouvaient être substitués à d'autres. Bref, je pense que les phénoménologues décrivent en réalité le plus souvent l'expérience "en troisième personne », i.e. à l'écart d'euxmêmes, sans s'impliquer de l'intérieur et sans être en contact ressenti avec ce qu'ils décrivent. Il y a là à mon sens une apparence d'objectivité et de neutralité, qui risque la généralité abstraite ${ }^{1}$. Je distingue de cette phénoménologie le plus souvent « en troisième personne », une phénoménologie "en première personne », que je nomme aussi expérientielle ou pratique, et qui, pour l'être effectivement, requiert une méthodologie précise et rigoureuse d'accès à l'expérience singulière du sujet. Plusieurs critères sont à vérifier pour être sûr d'être "en première personne »: 1) se référer à une expérience singulière, située dans le temps et dans l'espace ; 2) l'évoquer en se mettant en posture de la re-vivre, et non de s'en souvenir de façon intellectuelle ; 3) procéder à une description séquencée qui permette de suivre le fil temporel (début, pendant, fin) ; 4) réitérer l'évocation et la description pour affiner la segmentation temporelle et la granularité de la description; 5) réorganiser le temps de la description pour le mettre dans l'ordre de l'expérience telle qu'elle a été vécue ${ }^{2}$.

Par «langage de la première personne », j'entends l'attitude de celui ou celle qui parle ou écrit, son mode, sa façon, de telle sorte que le langage soit au plus près, au contact de l'expérience vécue. Ceci suppose notamment que la description fasse usage de termes immédiatement intelligibles, d'usage courant, et pas des termes qui font référence à un contexte non-immédiatement disponible. Par exemple, plutôt que de parler $\mathrm{d}^{\prime}$ " épochè », je décrirai l'expérience comme un moment de rupture avec le flux continu des impressions sensibles, de prise de recul, de questionnement de ce qui m'apparaît, etc. Bref, il ne s'agit pas d'un langage dont je ferai la doctrine ou la théorie, mais d'une pratique du langage, d'un usage en acte. Le terme

\footnotetext{
1. À ce propos, N. Depraz, "Self-givenness, first-person givenness and first-person method in Husserl ", Copenhagen Conference, oct. 2009, submitted in Phenomenology and the Cognitive Sciences.

2. Pour une première tentative de ce genre, N. Depraz, «La défaillance du sens. Premiers pas en direction d'une pratique phénoménologique en première personne ", in : Ten years of viewing from within, Cl. Petitmengin éd., JCS, 2009. Plus largement, «Qu'est-ce qu'une phénoménologie en première personne ? " in Première, deuxième, troisième personne, Colloque organisé par N. Depraz et A. Logeay les 14 et 15 mai 2009 à l’Université de Rouen, en préparation.
} 
«éloquence» pointe cet aspect modal et pratique du langage, sa dimension de ce fait communicative et d'adresse, en tout cas située ${ }^{3}$.

La question à laquelle je vais tenter de donner quelques éléments de réponse est la suivante : quels sont les critères d'un langage (de la) première personne? Où il s'agira, non pas de parler « de » la première personne (en extériorité et en surplomb, bref, en «troisieme personne »), mais, au fond, de parler «première personne ». Pour faire entendre « dans la langue » ce mode expérientiel incarné de relation aux mots, je vais procéder en trois temps, en adoptant une variation expressive linguistique, qui me permettra pas à pas de cerner plus avant le mode juste du contact expérientiel avec les mots, à distance des concepts généraux, souvent mots aux pouvoirs magiques ou cache-misère ! Je vais partir de l'expression la plus couramment utilisée pour cerner ce type de langage : "à la première personne », qui fait référence à l'énonciation linguistique et à l'usage du pronom personnel ; puis je m'intéresserai à la dimension méthodique en usage dans les sciences cognitives et, plus particulièrement en psychologie : «en première personne », qui porte l'accent sur le mode d'accès à l'expérience vécue singulière ; enfin, je chercherai à faire apparaître un langage de la première personne (non plus «à la », ni « en »), qui tente de montrer comment parler « première personne ».

\section{I. « À la » première personne}

Parler à la première personne, c'est dire Je (ou bien Nous) ${ }^{4}$, en tout cas, c'est parler en son nom propre, c'est-à-dire revendiquer ce que l'on dit comme sien et adhérer à ce que l'on dit.

L'expression «à la première personne » a un sens immédiatement grammatical et, plus avant, linguistique, exemplairement thématisé par E. Benvéniste dans les Problèmes de linguistique générale, ce qui le conduit à adopter une position radicale concernant l'importance du pronom personnel «Je » en tant que marqueur de la subjectivité en son sens ontologique : «qui dit Je est Je », énonce-t-il de façon univoque.

\footnotetext{
${ }^{3}$. À ce propos, N. Depraz, Ecrire en phénoménologue. Une "autre "époque de l'écriture, La versanne, Fougères, 1999 ; «L'épochè phénoménologique comme éthique de la prise de parole. Deux terrains pratiques: l'écriture poétique et l'intervention psychiatrique » in: Ethique du rapport au langage (sous la dir. de M. Castillo), Paris, L'Harmattan, 2007 ; Lire Husserl en phénoménologue. Les idées directrices I, Paris, PUF/CNED, 2008 ; «Lire et écrire en phénoménologue: Sartre et l'accès au vécu "en première personne" " in Lire et écrire: des phénomènes-miroir. $\grave{A}$ l'exemple de Sartre, Journée d'étude du 20 mars 2009 (org. par N. Parant et N. Depraz), Université de Rouen, publication en préparation.

4. À ce propos, N. Depraz, « Phénoménologie du "nous" », Alter no 17, oct. 2009.
} 
L'apriori du langage comme ce qui dicte l'être n'aura pas été affirmé plus nettement. Certes, "dire Je », dès l'instant où ce je est une instance de discours ou d'énonciation, c'est-à-dire se trouve inscrit dans une situation ou un contexte apparait nettement comme un «indexical essentiel », selon l'expression de J. Perry, qui porte en lui-même la subjectivité la plus intime de celui qui s'exprime, à savoir manifeste son implication personnelle et reflète l'adhesion à ce qu'il dit. C'est également le sens de l'hypothèse de S. Chauvier dans Dire Je. Essai sur la subjectivité.

Cependant, le pronom personnel Je, à lui seul, ne permet pas d'identifier une prise de parole incarnée et, ce, dans deux cadres au moins : 1) la tradition phénoménologique, portée ici essentiellement par Husserl, Sartre et M. Henry, met en avant un Je, un ego ou un soi/ipse qui s'annonce sur un mode générique, et reconduit pour une part essentielle une conception du sujet qui a toutes les apparences d'une entité métaphysique non-incarnée. Il ne s'agit que très rarement d'une instance d'énonciation répondant à une situation précise : le Je transcendantal est l'objet unique de cette science qu'est la phénoménologie, certes, mais il ne saurait être une "première personne", au sens d'un locuteur qui s'exprime en son nom propre et témoigne par exemple de ce qu'il vit; 2 ) dans le cas où l'on a bien affaire à une instance-locuteur, comme c'est le cas chez Shoemaker ou Perry, la caractéristique du « Je » est d'être auto-référentielle, mais, à ce titre aussi, d'être vide de contenu singularisant: chacun d'entre nous dit «Je », et il s'agit à chaque fois de moi-même : l'universalité de l'auto-référentialité du Je requiert, pour que ce Je devienne une première personne incarnée, que la situation permette de préciser, par la concrétude de l'action spatio-temporelle, qu'il s'agit bien, à chaque fois, de moi et de personne d'autre.

Ces deux cadres (phénoménologique/analytique) permettent de relativiser la primauté exclusive du pronom personnel Je comme emblématique de la "première personne ». De ce point de vue, l'expression linguistique " "à la" première personne » manifeste une exclusivité du langage qui le laisse en position d'extériorité partielle par rapport à l'entente «incarnée » de la première personne. Bref, le pronom personnel Je, à lui seul, le plus souvent ne suffit pas à définir le langage de la première personne, même s'il peut parfois en être l'indicateur.

\section{II. « En » première personne}

D'où l'importance de compléter la modalité linguistique «à la première personne » par une modalité expérientielle «en première personne ». De «à la » à «en », on passe d'une détermination langa- 
gière à une compréhension expérientielle. Mais cette dimension n'est pas pour autant de l'ordre du privé, de l'anecdotique, ni sauvage ou personnelle au sens des journées intimes, voire d'une forme existentielle d'introspection.

Parler "en première personne », c'est adopter, selon l'expression de P. Vermersch, une "prise de parole incarnée ", c'est-à-dire entrer dans un régime d'expression qui manifeste à plein la réalité concrète d'un contact avec ce que l'on vit au moment où je le formule. Le discours en fournit des indications, mais c'est le vécu qui permet d'en fournir le critère de fond. Par exemple, si je suis, au moment où je vous parle, en train de penser ce que je vous dis, mon débit de parole sera lent, voire ralentira, je chercherai mes mots, signe, non de nonassurance, mais de contact avec le vécu de la pensée en train de se formuler ; au contraire, si mon débit est très rapide, sans pause, c'est que je vous livre du " prêt à penser », qui correspond à une construction toute faite, et que je ne suis pas en train, au moment même, d'habiter, d'éprouver ou d'incarner.

Ce que l'on nomme aujourd'hui les « approches » ou «méthodes » " en " première personne ne se contentent pas, loin s'en faut, de souligner cette exigence $d$ 'une attestation vécue de la formulation langagière, à titre de support expérientiel effectif, même si l'on peut considérer que cela permet déjà de décentrer le langage de son apriorité et de se recentrer autour d'un vécu dont le langage n'est que le support.

Ces démarches, en jeu dans l'entretien d'explicitation puis d'autoexplicitation développées par P. Vermersch et, plus récemment, par $\mathrm{Cl}$. Petitmengin, insistent sur la nécessité d'une méthode précise, détaillée, rigoureuse, bref, disciplinée, d'accès à l'expérience singulière d'un sujet singulier. De façon un peu simplifiée ici, il s'agit de partir d’une expérience spécifiée (i.e. non « en général »), inscriptible dans un temps et un espace uniques, de l'évoquer sur le mode concret d'un re-vécu (i.e. de s'y replonger, de la revivre), et non de la formuler à distance de soi-même, en surplomb, comme un souvenir que j'observe de l'extérieur, puis de la deccrire en reprenant les différentes phases temporelles de son déroulement, enfin, de réitérer cette évocation/description le nombre de fois le plus important possible, jusqu'à épuisement des traits descriptifs disponibles. Ces quelques indications ne fournissent qu'un schéma très minimal de la méthode d'auto-explicitation, mais elles permettent déjà de mesurer qu'il s'agit d'un travail méthodique, qui prend du temps, et qu'il ne s'agit en rien d'une description spontanée, immédiate, bref, sauvage, de son expérience personnelle, comme, très souvent, on en trouve des exemples non-construits, très peu soigneux, dans les descriptions des philosophes, chez Sartre le premier, mais aussi chez Merleau-Ponty par exemple. 
Plus avant, la présence du « je » en tant que pronom personnel, est souvent un indicateur fiable d'une prise de parole incarnée, mais, comme on l'a noté, il peut également y avoir illusion d'incarnation. Comme l'a montré $C$. Petitmengin dans "At the source of thoughts » (JCS), la non-présence du je n'empêche pas, parfois, un mode de discours très en contact avec sa propre expérience, très relié, que je nomme de mon côté «l'intime » par distinction d'avec le "privé ». $\mathrm{Au}$ delà $\mathrm{du}$ «je » en tant que pronom personnel, ce qui est fondamental, c'est la présence à ce que l'on dit, le mode de relation concrète à ce qui est vécu, mode expérientiel qui peut très bien passer par le «on» ou par l'impersonnel de l'infinitif, ainsi que je viens de le mettre en scène.

Ces méthodes psychologiques issues de l'introspection mais lui conférant une rigueur et une granularité remarquables permettent de doter l'approche phénoménologique, souvent générique et sans lest expérientiel, d'une inscription concrète et méthodique. Pour autant, elles se situent sur le terrain d'une «méthode d'accès » à la première personne, dont on peut se demander si le caractère méthodique ne maintient pas une dichotomie un peu naïve encore entre la methode et l'être : le «en » de «en première personne » manifeste cet accent porté sur l'accès à ...

\section{III. « De » la première personne}

À cet égard, il y a encore trop d'éloquence dans le mode méthodique " en première personne », de même qu'il en avait de fait beaucoup trop dans le mode linguistique exclusif «à la première personne ». Il s'agit de prendre ces éloquences linguistiques et méthodiques et de leur «tordre le cou », comme disait Verlaine.

Pour autant, le langage « de » la première personne, si l'on adopte à présent ce régime, ce mode du "de ", n'est pas an-éloquent, n'est pas neutre ni seulement formel: il y a une force expressive de la première personne en parole ou en écriture. Quelle est en ce sens la «vraie éloquence » qui «se moque » d'une éloquence-artefact (linguistique ou méthodique)?

Le "de », en premier lieu, nous oriente en deçà du régime distinctif, qu'il soit celui de la dichotomie accès à.../objet, ou de la différence Je/non-Je. Deuxièmement, le langage de la première personne met au premier plan un critère de justesse expressive qui passe par une prudence dans l'énonciation et un mode de relation à soi-même et à l'expérience à décrire sans cesse en vérification de sa présence à moi-même. Husserl, au § 96 des Idées directrices I, fournit 
des indications précises de cette atttitude, qui correspond à une sorte d'éthique pratique du phénoménologue-locuteur ou scripteur ; troisièmement, un tel langage implique un usage des mots ordinaires plutôt que de concepts qui ne sont intelligibles qu'à partir de sources de références non-immédiatement disponibles. A cet égard, Wittgenstein dans sa dernière période, de par son insistance sur le langage ordinaire, est une ressource précieuse.

C'est pourquoi, j'ai choisi de mettre le «de» de mon intitulé "L'éloquence "de" la première personne » entre guillemets, en faisant jouer à ces derniers le rôle, souvent noté par Husserl lui-même, d'un opérateur tangible, inscrit dans la langue, de l'épochè : pourquoi une telle mise entre parenthèses $\mathrm{du}$ " de »? Le rôle grammatical immédiat de ce dernier, on l'a vu, est de faire de la " première personne » un objet sur lequel porte l'éloquence. On pourrait ainsi reformuler le titre en disant: "l'éloquence qui porte sur la première personne », ce qui confère au « de » le statut grammatical d'un regard sur, lequel ouvre un deuxième plan "méta», et crée de fait une dichotomie entre l'accès à... et l'objet. En suspendant ce mouvement d'écart et de dichotomisation, on entre directement dans le phénomène même du "langage première personne ", en adoptant une position d'immanence a l'objet, à la manière de Sartre qui, dans L'être et le néant, utilisait des crochets droits pour supprimer la médiation réflexive en jeu dans l'expression de la «conscience thétique [de] de soi ».

En conclusion, on peut dire que l'éloquence propre à la première personne tient dans la disparition des marqueurs linguistiques, méthodiques ou conceptuels, bref, dans le retrait des indicateurs de l'éloquence. La première personne apparaît d'autant plus que son marquage explicite s'efface. C'est me semble-t-il le sens profond de la "vraie éloquence » dont parle Pascal, soit de la vraie rhétorique, c'està-dire aussi de l'intimité effective. 